\title{
CARL SCHMITT LECTEUR DE VITORIA
}

\section{[Carl Schmitt reader of Vitoria]}

Céline Jouin*

Dans un article intitulé «Vitoria und die Geschichte seines Ruhmes », que Schmitt a publié anonymement en juillet 1949 dans la revue Die Neue Ordnung et qu'il a repris ensuite dans Le nomos de la terre en le modifiant légèrement, Schmitt donne son interprétation de l'œuvre de Francisco de Vitoria (1486-1546) et de sa place dans l'histoire du droit des gens. Depuis la fin de la Première Guerre mondiale, le théologien espagnol et défenseur du droit des Indiens était devenu le maître des pacifistes et les wilsoniens qui le présentaient comme le véritable père du droit des gens. Dans son journal, Schmitt note que «Vitoria et la dé-théologisation du droit des gens (Enttheologisierung des Völkerrechts) » est «son grand thème ${ }^{1}$ ». On s'en aperçoit en lisant le Glossarium². La critique de son article de 1949 sur Vitoria par l'internationaliste von der Heydte ${ }^{3}$ avait d'ailleurs beaucoup affecté Schmitt, qui n'a pas répondu publiquement à son collègue mais qui a longtemps exprimé son irritation à ses amis $^{4}$.

La lecture que Schmitt délivre des Relectiones de Vitoria a beau être fameuse, elle est rarement commentée et ceux qui tentent de l'interpréter expriment souvent le désarroi dans lequel elle les laisse ${ }^{5}$. Certains spécialistes qui sont d'accord avec Schmitt

\footnotetext{
* Maître de Conférences en philosophie à l'Université de Caen Normandie depuis 2011, celinejouin@gmail.com.

${ }^{1}$ Carl Schmitt, Glossarium. Aufzeichnungen der Jahre 1947-1951, Berlin, Duncker \& Humblot, 1991, p. 25.

${ }^{2}$ Glossarium, op. cit., p. 105-106, p. 173, p. 266.

${ }^{3}$ Friedrich August Freiherr von der Heydte, «Francisco de Vitoria und die Geschichte seines Ruhmes - Eine Entgegnung », Die Friedens-Warte, n4/5, 1949, p. 190-197.

${ }^{4}$ Voir la lettre de Carl Bilfinger à Schmitt du 19 décembre 1949, Archives Carl Schmitt, Hauptstaatsarchiv de Düsseldorf, RW 265-1385 ainsi que le Glossarium, op. cit., p. 276.

5 À notre connaissance, après von der Heydte, c'est Wilhelm Janssen qui est allé le plus loin dans l'interprétation de la lecture schmittienne de Vitoria, qu'il réinscrit dans l'ensemble des débats sur Vitoria des internationalistes du XX $\mathrm{XX}^{\mathrm{e}}$ siècle, à partir d'Ernest Nys . Cf. Wilhelm Janssen, Die Anfänge des modernen Völkerrechts und der neuzeitlichen Diplomatie. Ein
} 
pour dénoncer le contresens des pacifistes sur Vitoria ne se réclament pas de $\mathrm{Schmitt}^{6}$; d'autres considèrent que c'est Joseph Höffner (Christentum und Menschenwürde, 1947), et non Schmitt, qui, dans l'après guerre, a donné l'interprétation la plus pénétrante de la Seconde scolastique et du sens qu'elle a pour le droit international ${ }^{7}$.

Notre interprétation de la lecture schmittienne des Relectiones repose sur deux hypothèses :

1/ La première a un caractère philologique. On sait que Le nomos de la terre n'a pas été rédigé d'une seule traite. Schmitt a composé son livre en reprenant divers articles qu'il avait écrits à la fin des années 1930 et au début des années 1940. Il procédait en «plaçant » dans son livre des thèmes et des textes auxquels il tenait ${ }^{8}$. Il semble qu'il y ait deux ensembles hétérogènes de textes qui concernent Vitoria dans Le nomos de la terre, textes écrits à des moments différents : le long chapitre que le juriste consacre au dominicain espagnol ${ }^{9}$ reprend, en ne le modifiant que légèrement, son article paru en 1949 sur Vitoria («Vitoria und die Geschichte seines Ruhmes »). Il s'agit d'un texte unitaire, que nous appellerons le «chapitre sur Vitoria». Mais Schmitt parle également de Vitoria en d'autres lieux du Nomos de la terre (que nous appellerons «le second groupe de textes »), dans des passages qui ont une grande signification parce qu'ils ont la double fonction de situer Vitoria par rapport aux autres auteurs classiques, comme Grotius et Pufendorf, et de rattacher l'article de 1949 sur Vitoria à l'architecture d'ensemble du Nomos de la terre, opération, nous le verrons, qui échoue en partie : il s'avère que les conclusions du « chapitre sur Vitoria » et celles du «second groupe de textes » sont peu compatibles les unes avec les autres. Dans Le nomos de la terre, le cinquième corollaire introductif intitulé «La prise de terres comme événement constituant du droit des gens ${ }^{10} \gg$ et la section portant sur Grotius et Pufendorf ${ }^{11}$ sont les deux moment clés de ce second groupe de textes. Schmitt n'a l'air d'y parler de Vitoria qu'en passant, mais une lecture attentive révèle que l'ensemble du développement sur

Forchungsbericht, Stuttgart, J. B. Metzlersche Verlagsbuchhandlung, 1965, p. 2-14 et p. 55-59.

${ }^{6}$ Voir Arthur Nussbaum, «James Brown Scotts Thesen über die Überlegenheit der spanischen Scholastiker über Hugo Grotius », in Geschichte des Völkerrechts in gedrängter Darstellung, Munich, Berlin, Beck, 1960 [1947], p. 327-339.

7 Voir Wilhelm Janssen, Die Anfänge des modernen Völkerrechts und der neuzeitlichen Diplomatie, op. cit., p. 53.

${ }^{8}$ Voir Glossarium, op. cit., p. 141.

${ }^{9}$ Carl Schmitt, Le nomos de la terre dans le droit des gens du Jus publicum Europaeum, trad. par Lilyane Deroche-Gurcel et Peter Haggenmacher, Paris, Presses Universitaires de France, 2001, p. 102-126.

${ }^{10}$ Le nomos de la terre, op. cit., p. 85.

${ }^{11}$ Ibid., p. 134-139. 
Grotius et Pufendorf est en fait un portrait indirect de Vitoria - qui est le point de comparaison constant de Schmitt quand il parle des deux juristes. Les mots suivants expriment bien la structure de cette section : «Aucun des deux [Grotius et Pufendorf] ne traite la question clé, la prise territoriale du sol non européen par les européens [...] tandis que Vitoria garde en vue le problème central ${ }^{12} »$.

2/ Notre seconde hypothèse a un caractère plus général. Elle ne concerne pas seulement Vitoria, il ne s'agit que de la vérifier à propos de Vitoria. L'hypothèse est que la théorie de l'impérialisme d'auteurs comme Lénine ou Rosa Luxemburg est le « centre absent » d'une série de textes de Schmitt et que le juriste applique cette théorie aux lieux communs de la doctrine du droit international pour la rajeunir mais aussi pour la critiquer, pour montrer que par certains aspects, elle a un caractère dérivé. Or, dans le cas qui nous occupe actuellement, cette application est visible uniquement dans le « second groupe de textes ».

Au départ, le chapitre sur Vitoria du Nomos de la terre est une longue protestation contre la «réutilisation » de Vitoria par les pacifistes et les wilsoniens. Ce culte du théologien espagnol, les spécialistes considèrent aujourd'hui qu'il était effectivement fondé sur une série de contresens et sur une idéologie bien spécifique, que Wilhelm Janssen nomme «l'idéologie de la SDN» (Völkerbundsideologie $\left.{ }^{13} »\right)$. Depuis la Première Guerre mondiale, la renaissance de Vitoria participait d'une reviviscence du droit naturel, au moment de la fondation de la SDN. Cette ferveur pour les Relectiones participait aussi - cet aspect est plus rarement souligné - de l'émergence de l'histoire du droit international comme genre scientifique. Le principal responsable de ce que Schmitt appelle tantôt le «mythe politique » tantôt le «mythe journalistique » de Vitoria (NT: 118) est l'internationaliste américain James Brown Scott (1866-1943), promoteur de l'histoire du droit international et directeur d'un grand nombre de centres de recherches, comme l'American Institute of International Law ou l'American Society of International Law. La renaissance de la seconde scolastique dans l'entre-deuxguerres doit beaucoup à la grande influence qu'exerçait l'internationaliste américain sur l'opinion publique américaine et mondiale. Ernest Nys est le premier à avoir tiré le nom de Vitoria de l'oubli où il était tombé à la fin du XIX ${ }^{\mathrm{e}}$ siècle $^{14}$. James Brown Scott a pris

\footnotetext{
12 Ibid., p. 138. Nous soulignons.

${ }^{13}$ Wilhelm Janssen, ibid., p. 51.

14 Voir Ernest Nys, Le droit de la guerre et les précurseurs de Grotius, Bruxelles, Leipzig, C. Muquart, 1882 ; Ernest Nys, Les origines du droit international, Bruxelles, Castaigne, 1894 ; James Brown Scott, The Spanish Origin of International Law. Francisco de Vitoria and his Law
} 
la suite des remarquables travaux du juriste belge, mais il a traité la pensée du dominicain avec moins de précautions que lui. Peu à peu c'est l'interprétation de Scott qui a pris le dessus, sans être soumise à une discussion scientifique sérieuse, parce qu'elle permettait mieux que celle de Nys de légitimer les nouvelles institutions internationales et la politique extérieure américaine.

Des historiens du droit comme Arthur Nussbaum ou Peter Haggenmacher ont livré le même diagnostic sévère que Schmitt à l'endroit de James Brown Scott. Schmitt n'est pas le seul à accuser le juriste américain de superficialité. Scott suivait une ligne politique claire. Il avait plaidé pour la poursuite pénale de Guillaume II à la Conférence de Paris de 1919. Pour Schmitt, il symbolisait le retour de la doctrine de la guerre juste : les guerres à venir se mettront de plus en plus à ressembler aux guerres de religions qui ont déchiré l'Europe avant que l'État moderne ne les jugule, mais il faut ajouter que ce n'est qu'une ressemblance approximative, car il n'y a pas de retour en arrière dans l'histoire, ce dont Schmitt était conscient. La théorie de la guerre juste qui était selon Schmitt au fondement du Protocole de Genève du 2 octobre 1924, mais aussi de la Conférence du désarmement de Genève de 1932 - 1934 et du pacte Briand-Kellogg de 1928, n'avait plus rien à voir avec l'ancienne doctrine médiévale. Les doctrinaires contemporains de la guerre juste invoquaient à tort, selon Schmitt, les théologiens de la Seconde scolastique pour soutenir leurs thèses.

James Brown Scott affirmait que Vitoria « avait décrit explicitement l'institution des mandats telle qu'elle existait au sein de la Société des Nations ${ }^{15}$ » ainsi que la politique américaine de la porte ouverte. Selon lui Grotius s'était contenté pour une grande part de reprendre la pensée de Vitoria ${ }^{16}$. En outre, Scott traduisait le jus gentium de Vitoria par international law, mêlant continûment les problèmes contemporains à la pensée du théologien espagnol.

On peut relever les deux thèses suivantes dans la réponse que Schmitt fait à James Brown $\operatorname{Scott}^{17}$ :

1/ La première objection qu'il adresse au juriste est la thèse selon laquelle Vitoria n'est pas un précurseur du droit des gens interétatique moderne mais qu'il reste attaché à la conception médiévale du jus gentium. Il invite à prêter une grande attention à la

\footnotetext{
of Nations, Londres, Milford, 1934.

15 J. B. Scott, The Catholic Conception of International Law, Washington, Georgetown University Press, 1934, p. 31.

${ }^{16}$ Id., The Spanish Origin of International Law, op. cit., p. 336.

${ }^{17}$ Le nomos de la terre, op. cit., p. 102-126.
} 
différence des contextes ${ }^{18}$. Sous le nom de jus gentium les Romains entendaient non pas des rapports entre peuples (inter gentes) mais les normes de «droit privé » qui réglaient les relations individuelles entre les citoyens romains et les non Romains, ou des non Romains entre eux. Les rapports juridiques entre citoyens romains étaient en revanche régulés par le jus civile. Le Moyen Âge a étendu le sens romain : le jus gentium médiéval était un droit valable pour tous les peuples, il incluait au surplus le droit de la guerre et de la paix, et non seulement le droit civil. Isidore de Séville, au seuil de la pensée médiévale, a essayé de concilier le sens étroit de l'Antiquité et le sens large du Moyen Âge. Schmitt le mentionne ${ }^{19}$. James Brown Scott commet un contresens quand il fait de Vitoria le père de la SDN car le dominicain espagnol conçoit le jus gentium comme les autres auteurs du Moyen Âge. C'est Suarez après lui (1548-1617) qui sera le premier théoricien du droit interétatique moderne et qui définira le jus gentium comme un jus inter gentes, un droit interétatique, au sens qui est le nôtre. Sur ce point, la plupart des spécialistes, aujourd'hui, sont d'accord.

Mais Schmitt n'en reste pas à cet argument. Il ne se contente pas de souligner la différence entre les juristes du $\mathrm{XX}^{\mathrm{e}}$ siècle et les théologiens scolastiques. Il expose une thèse plus extrême et plus problématique, par laquelle il rejoint Christentum und Menschenwürde (1947) du futur cardinal Joseph Höffner, livre paru quelques années avant Le nomos de la terre et qui, avec les textes de Truyol y Serra, est l'ouvrage qui a le plus marqué la recherche sur Vitoria du second après-guerre. À l'instar d'Höffner, Schmitt soutient que l'horizon idéal de la théorie de Vitoria n'est pas l'humanité entière (totus orbis) mais l'Église chrétienne, Église dont l'idéal est, certes, de s'étendre sur tout le globe (una ecclesia, Weltkirche), mais qui est en premier lieu lié à un espace concret et à une civilisation particulière. «L'objectivité et la neutralité de Vitoria n'en ont pas moins leurs limites et ne vont pas du tout jusqu'à lui faire réellement ignorer et nier la différence entre chrétiens et non-chrétiens. Au contraire : le résultat pratique est entièrement fondé sur la foi chrétienne de Vitoria qui voit dans la mission chrétienne la véritable justification de la conquête ${ }^{20} »$. «À cet égard, l'Europe chrétienne est donc encore bien le centre de la terre pour Vitoria ${ }^{21} »$. Alors que la majorité des spécialistes

18 Voir aussi le texte inédit de Schmitt intitulé Entwicklung der internationalrechtlichen Pönalisierung des Angriffskrieges (1919-1939), Hauptstaatsarchiv de Düsseldorf, RW 26519220, p. 48.

${ }^{19}$ Le nomos de la terre, op. cit., p. 49.

${ }^{20}$ Le nomos de la terre, op. cit., p. 114.

${ }^{21}$ Ibid., p. 115. 
s'accordaient à reconnaître le premier point - la dépendance de Vitoria par rapport au jus gentium médiéval - sur ce second point, les avis divergent. Höffner et Schmitt sont accusés $^{22}$ de sous-estimer la portée universaliste de la pensée du théologien espagnol et l'importance chez lui du totus orbis. On leur objecte que le droit des gens médiéval était bel et bien universaliste, que l'idée d'unité du monde et l'idée de Chrétienté ne s'excluaient pas mutuellement. Contrairement à ce que Schmitt affirme, elles étaient tout à fait compatibles, étant toutes deux au fondement du jus gentium. La Chrétienté n'était pas un «grand espace de droit international» fermé aux interventions des étrangers comme Schmitt cherche à le faire croire. Pour cela, le juriste va jusqu'à identifier la guerre juste telle que la conçoit la Seconde scolastique aux croisades contre les infidèles ${ }^{23}$, alors qu'il sait que la guerre juste implique la limitation de la guerre. La guerre juste suppose en effet la présence d'un justus hostis, une intention droite, la proportionnalité entre les moyens employés et la violence initiale et une procédure ordonnée, caractéristiques qui sont absentes de l'idée de croisade. Schmitt le sait, il le rappelle à l'occasion ${ }^{24}$.

À partir de là, une question se pose: si le professeur de Salamanque s'inscrit pleinement dans la pensée médiévale et théologique, pourquoi est-il «le grand thème » de Schmitt, comme le juriste l'écrit lui-même dans son journal ${ }^{25}$ ? Plus précisément, pourquoi Schmitt affirme-t-il dans le Glossarium que son «grand thème » est Vitoria «et la dé-théologisation du droit des gens », s'il le rattache d'autre part avec insistance à l'univers de la théologie médiévale ?

On sait que la question s'était politisée après la Première Guerre mondiale. Ceux qui se réclamaient de Vitoria étaient surtout des Anglo-Saxons et des Américains qui affirmaient que le Jus publicum europaeum sécularisé et son jus ad bellum représentaient un déclin par rapport à «l'origine », identifiée à la théorie médiévale de la guerre juste. Schmitt pensait que le droit des gens sécularisé apparu au XVI ${ }^{\mathrm{e}}$ siècle

\footnotetext{
${ }^{22}$ Voir Wilhelm Janssen, op. cit., p. 55 et Friedrich August von der Heydte, op. cit., p. 194.

${ }^{23}$ «Les croisades et les guerres de mission autorisées par l'Église étaient par là même des guerres justes, indépendamment de leur caractère offensif ou défensif ; les princes et les peuples qui persistaient à refuser l'autorité de l'Église, comme les Juifs et les Sarrasins, étaient par là même hostes perpetui », Le nomos de la terre, op. cit., p. 120-121.

${ }^{24}$ Il écrit par exemple en 1938 dans «Das neue Vae Neutris!», in Positionen und Begriffe im Kampf mit Weimar-Genf-Versailles, 1923-1939, Berlin, Duncker \& Humblot, 1994 [1940], p. 289: "guerre juste n'est pas guerre sainte», et dans Ex captivitate Salus. Expériences des années 1945-1947, trad. par André Dorémus, Paris, Vrin, 2003, p. 152 : «il y a des guerres justes, des guerres saintes, et des guerres en forme de duel ».

${ }^{25}$ Glossarium, op. cit., p. 25
} 
était un progrès par rapport au Moyen Âge. On comprend qu'il ait voulu « reprendre » Vitoria aux pacifistes et aux wilsoniens. S'il accorde un long chapitre à Vitoria dans son histoire - somme toute assez courte - du droit international, c'est qu'il s'agissait de ne pas laisser Vitoria aux pacifistes. On peut d'ailleurs noter que dans le débat qui faisait rage vers la Première Guerre mondiale (est-ce Grotius ou Vitoria, le père du droit international ?), Schmitt a ceci en commun avec les pacifistes et les libéraux qu'il accorde une grande importance à Vitoria et déprécie Grotius. Les choses sont donc passablement compliquées : en somme, Schmitt fait l'éloge de la sécularisation du droit des gens et de l'aspect procédural de la guerre, tout en donnant au théologien qu'est Vitoria une place éminente dans l'histoire du droit des gens et en refusant de reconnaitre que la laïcité et l'humanité deviennent avec lui les horizons du droit. On est alors conduit à se poser la question suivante : si Schmitt juge positivement la sécularisation du droit international, pourquoi n'a-t-il pas porté comme d'autres toute son attention sur Suarez, plutôt que sur Vitoria, puisque c'est Suarez, en effet, qui suit de quelques décennies Vitoria, qui est le véritable père du jus inter gentes au sens du droit interétatique moderne?

2/ L'autre thèse insistante par laquelle Schmitt répond à Scott, controversée parmi les spécialistes, est que les Relectiones, notamment les deux leçons que le théologien de Salamanque a prononcées en 1538 et 1539, Sur les Indiens et Sur le droit de guerre, offrent une «justification de la prise territoriale d'un nouveau monde ». C'est le titre de son chapitre sur Vitoria. Ailleurs dans Le nomos de la terre, Schmitt a plutôt l'air de dire que la pensée de Vitoria a eu pour effet de légitimer la Conquista, sans que Vitoria en ait eu l'intention ou qu'il l'ait expressément justifiée lui-même ${ }^{26}$. Le juriste renverse la représentation commune qui veut que Vitoria ait été avec Las Casas le défenseur du droit des Indiens et l'adversaire de Sepúlveda. L'historiographe de Charles Quint a affronté Las Casas à Valladolid après la mort de Vitoria lors d'une dispute célèbre. Il s'appuyait sur Aristote pour dire que les Indiens sont par nature des esclaves. Vitoria n'est pas d'accord avec cette idée. Pour lui, même infidèles, «les Indiens sont des hommes, et non des singes », ce qui leur assure des droits. Il ne reconnaît à l'Empereur et à l'Église aucun droit de domination directe sur les terres d'Amérique. Historiquement cependant, il est vrai que Vitoria et Las Casas avaient pris parti pour le

\footnotetext{
${ }^{26}$ «La théorie de la guerre juste de Vitoria permet par exemple la prise territoriale de sol étranger non libre», Schmitt, Le nomos de la terre, op. cit., p. 85 ; «La vieille doctrine de la guerre juste dont le résultat essentiel était de procurer un titre juridique pour une prise de terres... », ibid., p. 170.
} 
pouvoir central espagnol et le monopole de la Couronne contre les autres puissances qui cherchaient à tirer leur part du butin ${ }^{27}$. Le combat de Las Casas et la théorie de Vitoria ont de fait abouti à « aménager l'oppression des Indiens sans pour autant la supprimer ${ }^{28}$ » au moment où l'empire espagnol faisait sa fortune de l'or des Amériques.

Schmitt semble embarrassé. Cherchant de toute évidence à briser «l'invention de tradition» des pacifistes et des wilsoniens, il lui faut rompre la filiation qui fait remonter le système des mandats de la SDN à Las Casas et à Vitoria. Mais la lettre des Relectiones, dans la forme où elles nous sont parvenues, ne lui permet pas d'aller droit au but : ce sont des notes d'étudiants qui adoptent la forme scolaire de l'époque et juxtaposent le pour et le contre sans trancher, comme s'il s'agissait d'exercices d'argumentation : aux titres légitimant la Conquista Vitoria oppose les titres qui ne permettent pas de la légitimer, sans donner d'avis final. L'alternative devant laquelle se trouve Schmitt est claire : si la protection du droit des «indigènes » est la pierre de touche du système des mandats de 1919, système défini par l'article 22 du Pacte de la SDN, alors on peut en effet en faire remonter l'origine à Las Casas et à Vitoria. Les wilsoniens et James Brown Scott l'affirment. Ils font du mouvement pour l'abolition de l'esclavage une étape entre Vitoria et la SDN. Leur histoire du droit international est l'histoire linéaire de l'humanisation de la guerre. Toutefois, si la pierre de touche du système des mandats n'est pas la protection des populations colonisées mais l'appropriation des matières premières et des marchés, les choses changent ${ }^{29}$. Schmitt veut détruire les illusions progressistes des libéraux mais le texte des Relectiones ne se laisse pas tordre à volonté. Il admet que Vitoria présente deux séries antithétiques de titres, les titres légitimes et les titres illégitimes, permettant ou bien de justifier la Conquista ou bien de déclarer son iniquité. Il reconnaît que les propositions de Vitoria ont la forme de raisonnements hypothétiques («si...alors»), que le théologien ne présente aucune synthèse dans ce balancement du pour et du contre : «il ne tranche pas sur le mode d'une sentence judiciaire », mais reste « à distance des faits » (NT:110). Il est vrai qu' «à l'aide de telles propositions générales et d'argumentations possibles, on

27 Voir Pierre-François Moreau, «Nature, culture, histoire », in François Châtelet et Gérard Mairet (éd.), Histoire des idéologies, vol. 3, Paris, Hachette, 1978, p. 34 ; Jörg Fisch, Die europäische Expansion und das Völkerrecht. Die Auseinandersetzungen um den Status der überseeischen Gebiete vom 15. Jahrhundert bis zur Gegenwart, Stuttgart, Steiner, 1984, p. 211. ${ }^{28}$ Pierre-François Moreau, ibid., p. 34.

${ }^{29}$ Sur les «inventions de tradition » successives des juristes et des hommes politiques déniant la nouveauté du système des mandats de 1919 et du système des trusteeships de 1945, voir Ramendra Nath Chowdhuri, International Mandates and Trusteeship Systems. A Comparative Study, La Haye, Nijhoff, 1955. 
parvient tout à fait à justifier dans l'ensemble la Conquista espagnole. Mais il s'agirait alors avant tout d'une question de fait et de situation, que le scolastique laisse ouverte sans y répondre. » C'est ce qui explique d'ailleurs, selon Jörg Fisch, que l'œuvre de Vitoria ait été l'objet de tant de discussions, les partisans comme les adversaires de la domination espagnole pouvaient y puiser les arguments qu'ils voulaient ${ }^{30}$.

Une question se pose alors : qu'est-ce qui pousse Schmitt à annoncer dans le titre de son chapitre sur Vitoria ${ }^{31}$, ce qu'il répète à plusieurs reprises ${ }^{32}$, que l'œuvre du théologien de Salamanque justifie la prise des Amériques par les Européens s’il ne le déduit pas du texte des Relectiones ? Pourquoi Schmitt éprouve-t-il le besoin d'interpréter Vitoria de cette façon ${ }^{33}$ ?

Force est de constater que la place que Schmitt réserve au théologien de Salamanque est bien la place de l'origine, comme chez les pacifistes et les libéraux. Mais l'origine n'a plus chez Schmitt le sens qu'elle avait pour eux : l'origine du droit des gens est pour le juriste la prise violente du sol non européen et non les normes du droit humanitaire. Toutefois, ce n'est pas ce que Schmitt dit tout le temps : cette thèse n'apparaît que dans le second groupe de textes et non dans le chapitre qui porte sur Vitoria. La raison à cela n'est pas impossible à deviner : il y a un véritable coup de force à faire de Vitoria le justificateur de la prise violente des Amériques. Ce n'est donc pas l'argument qu'il met au premier plan dans son chapitre sur Vitoria, celui-ci ayant avant tout pour objectif de répondre aux abus interprétatifs des wilsoniens par l'exactitude exégétique.

C'est ici que la division des textes schmittiens sur Vitoria en deux groupes devient utile. Nous avons dit que nous repérions dans Le nomos de la terre deux ensembles de textes sur Vitoria dont la date de composition et la visée différaient. Dans un premier temps, dans son chapitre sur Vitoria ${ }^{34}$, composé en premier et publié à part en 1949 , Schmitt cherche à débarrasser des fausses retouches l'image qu'on se fait de Vitoria ${ }^{35}$. Il veut montrer le «vrai» Vitoria, dissoudre les contresens «superficiels» et «journalistiques » diffusés par James Brown Scott. «Contre ces possibilités tragiques

\footnotetext{
${ }^{30}$ Voir Fisch, op. cit., p. 212.

${ }^{31}$ Le nomos de la terre, op. cit., p. 102.

${ }^{32}$ Ibid., p. 85, p. 170.

${ }^{33}$ C'est la question que F. A. von der Heydte pose à juste titre dans « Francisco de Vitoria und die Geschichte seines Ruhmes - Eine Entgegnung », op. cit., 1949, p. 196. La perspective de von der Heydte est très critique, mais même pour les commentateurs mieux intentionnés à l'égard de Schmitt, la lecture schmittienne de Vitoria paraît alambiquée. Voir par exemple Janssen, op. cit., p. 5.

${ }^{34}$ Le nomos de la terre, op. cit., p. 102-126.

${ }^{35}$ Voir ibid., p. 126.
} 
de réutilisations hétérogènes ${ }^{36} »$, le juriste rattache alors le théologien espagnol au champ médiéval, qu'il présente comme un ensemble d'ordres concentriques : l'ordre des dominicains, l'ordre européen, l'Église. Il met alors en avant la «méthode anhistorique $^{37}$ » de Vitoria, «l'absence » chez lui « de toute perspective historique ». Il souligne qu'on ne trouve « rien du tout sur la civilisation» au sens moderne dans les Relectiones $^{38}$. Il souligne par là même la distance qui sépare Vitoria des notions évolutionnistes modernes de progrès et de civilisation ${ }^{39}$, mettant en garde contre la tentation de confondre la théorie de Vitoria avec un Kulturkampf bismarckien ou une laïcisation à la française ${ }^{40}$.

Dans le second groupe de textes toutefois, c'est une tout autre image de Vitoria qui se dégage. Dans la section sur Grotius et Pufendorf du Nomos de la terre ${ }^{41}$, Schmitt reproche à Grotius de maintenir la question de «l'acquisition de la propriété » dans le cadre anhistorique du droit civil ${ }^{42}$. Précédemment Schmitt avait déjà affirmé à propos de la longue controverse sur la liberté des mers que «la pratique [...] et les déclarations officielles manqu[aient] d'un nouveau principe et de la clarté de concepts repensés à neuf ${ }^{43} \ldots$... et que «la discussion doctrinale au XVI ${ }^{\mathrm{e}}$ et $\mathrm{XVII}^{\mathrm{e}}$ siècles [...] n'a[vait] pas encore pris conscience des vrais problèmes ${ }^{44} \gg$. Plus tard, on comprend quel est ce « nouveau principe » qu'il reproche à Grotius et Pufendorf de ne pas avoir établi : ceuxci n'ont pas «établi de lien entre leur conception du droit de propriété originaire et la prise territoriale tout à fait concrète du sol non européen par les puissances européennes qui s'est déroulée sous leur yeux à une échelle gigantesque ${ }^{45}$ ». Schmitt regrette alors que Grotius et Pufendorf «maintiennent le jus gentium dans l'équivoque produite par la transformation de notions de droit civil romain en des généralités de droit naturel ${ }^{46} \gg$. «Le juriste étatique [du XVII ${ }^{\mathrm{e}}$ siècle] ne parvenait même plus à aborder la question clé, à savoir la prise territoriale de sol non européen [...]. Les distinctions capitales manquaient à la science du droit des gens d'alors, qui, depuis Grotius et Pufendorf, n'a

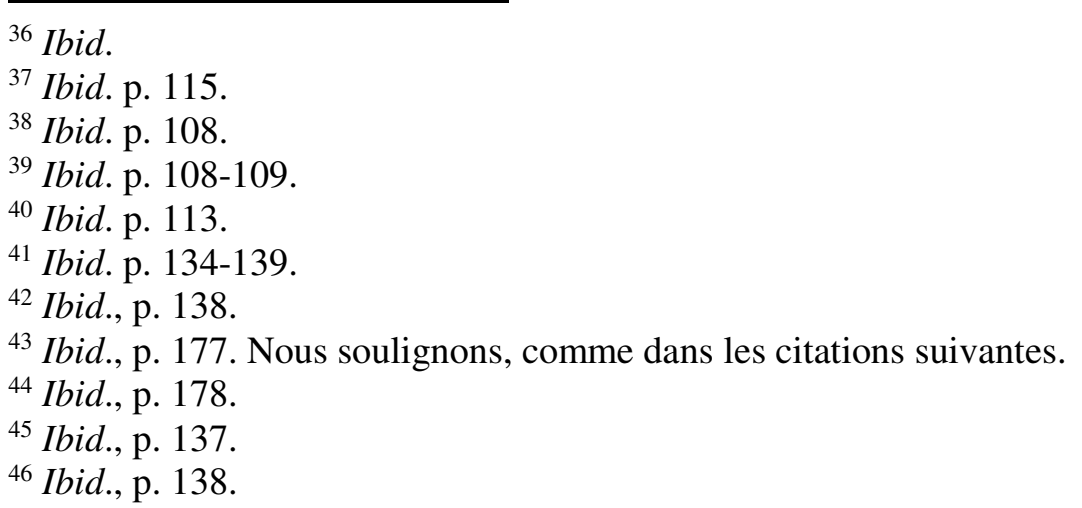


plus traité en termes juridiques des différences du statut du sol. Une telle science $d u$ droit des gens ne pouvait plus garder conscience de ses propres prémisses historiques $^{47}$ ». Et plus loin : «Les juristes du droit des gens [des XVII ${ }^{\mathrm{e}}$ et $\mathrm{XVIII}^{\mathrm{e}}$ siècles] restèrent prisonniers d'une perspective intra-européenne et ne virent presque jamais que l'ordre du Jus publicum europaeum était déjà global ${ }^{48}$ »; «les développements internationalistes des deux célèbres juristes [Grotius et Pufendorf] ne touchent qu'à des controverses secondaires et passent à côté de la structure concrète du droit des gens européen d'alors ${ }^{49} »$. Schmitt martèle toujours la même idée : Vitoria est le bon élève de son histoire. "Tandis que Vitoria garde encore en vue le problème central - la légitimité de principe de la prise territoriale du sol américain en tant qu'opération jure gentium -, ces prétendus fondateurs du droit des gens modernes ne parlent jamais que de l'acquisition en général $^{50} »$.

Par une série de glissements adroits, Schmitt a rangé Vitoria du côté de «la science $d u$ droit des gens qui garde conscience de ses propres prémisses historiques ». On est donc loin de la «méthode anhistorique » et «à distance des faits » que Schmitt attribuait à Vitoria une dizaine de pages plus haut dans le chapitre qu'il lui consacrait, chapitre qui partait d'une analyse détaillée du texte des Relectiones et de leur structure consistant à opposer le pour et le contre sans trancher.

Analysons un dernier extrait du second groupe de textes. Dans le cinquième corollaire introductif, le corollaire fondamental sur la prise de terres (Landnahme), le nom d'un seul grand juriste est invoqué : celui de Vitoria ${ }^{51}$. Il est clair que pour Schmitt, Vitoria est «le » penseur de la prise de terres et n'oublions pas que la prise de terres est pour lui «l'événement constituant du droit des gens ». Pourtant dans son chapitre sur Vitoria, chapitre qui se situe plus loin dans l'ouvrage mais qui a été écrit plus tôt, dans lequel il restait proche du texte des Relectiones et déployait face à Scott une «stratégie de l'exactitude », il avait été forcé d'admettre que, contrairement à la conscience juridique de son époque, Vitoria ne reconnaissait pas la découverte comme titre d'acquisition légitime ${ }^{52}$. En réalité, c'est Schmitt et non Vitoria qui affirme, ailleurs

\footnotetext{
${ }^{47}$ Ibid., p. 135.

${ }^{48}$ Ibid., p. 136-137.

${ }^{49}$ Ibid., p. 137.

${ }^{50}$ Ibid. p. 139.

${ }^{51}$ Ibid., p. 85.

${ }^{52}$ Ibid., p. 106.
} 
dans le livre, que la découverte est «le véritable titre juridique européen ${ }^{53}$ ». Dans son chapitre sur Vitoria, qui suit de très près le texte du théologien, Schmitt est forcé d'admettre que Vitoria ne justifie la conquête «que de façon médiate », par le biais du mandat de mission pontifical et du concept de guerre juste, qui lui, impose une foule de restrictions aux conquérants ${ }^{54}$ et qui est loin d'être un «chèque en blanc » tendu aux conquérants.

L'aveu par lequel Schmitt ouvre son cinquième corollaire est décisif : le juriste reconnaît en effet qu'il transforme le vocabulaire classique du droit des gens, et même le vocabulaire de la discipline moderne du droit international, quand il introduit le terme de Landnahme (prise de terres). Ni pour les juristes du $\mathrm{XX}^{\mathrm{e}}$ siècle ni pour Vitoria la notion de Landnahme n'est un lieu commun : l'expression n'est d'ailleurs pas courante en allemand, comme le fait remarquer Peter Haggenmacher. C'est le terme de LandTeilungen (partages de terre) qui est plus courant ${ }^{55}$. Schmitt mentionne «qu'il n'y a pour l'instant qu'une seule monographie d'histoire du droit qui fasse la synthèse sur la prise de terres » accomplie par les peuples germains à l'époque des grandes Invasions ${ }^{56}$. La «prise de terres » est donc loin d'être une catégorie générale de la science du droit. C'est le même diagnostic que font plus près de nous les historiens du droit Jörg Fisch et Martti Koskenniemi : les livres sur ce sujet sont rares, mais parce que la catégorie manque, non les matériaux. Si Fisch et Koskenniemi ajoutent quelques titres de livres à celui qu'indique Schmitt, c'est sans manquer de noter la rareté extraordinaire des études sur un sujet qui nous paraît aujourd'hui essentiel ${ }^{57}$.

En bref, Schmitt fait de Vitoria le théoricien de la prise de terres coloniales alors que l'auteur des Relectiones ne reconnaît pas la découverte comme titre juridique et bien que le concept de «prise de terres » ne se trouve pas chez lui. N'y a-t-il pas quelque chose d'étrange dans ce cas à faire de Vitoria le théoricien du «pouvoir constituant » du droit des gens par opposition à Grotius, Pufendorf et Gentili, qui deviennent du même coup les théoriciens des «institutions constituées $^{58} »$ ? On l'aura compris, il nous semble que le juriste allemand fasse de Vitoria le fondateur de la science du droit

${ }^{53}$ Ibid., p. 131.

${ }^{54}$ Ibid., p. 112.

${ }^{55}$ Ibid., p. 84.

${ }^{56} \mathrm{Ibid}$.

57 Jörg Fisch, Die europäische Expansion und das Völkerrecht, op. cit., p. 34, p. 157. Par exemple Fisch remarque l'absence de monographies exhaustives sur le rapport juridique des Européens avec les « infidèles », ou sur l'histoire de l'acquisition violente de territoires et sur le droit qui lui est lié.

${ }^{58}$ Ibid., p. 85. 
international comme elle devrait être et non comme elle est : c'est son programme du droit international comme «théorie de l'impérialisme appliquée » que Schmitt projette sur la pensée du professeur de Salamanque, programme d'une histoire du droit colonial, ou mieux : d'une histoire qui prendrait comme objet la relation entre droit colonial et droit européen et accorderait au second un statut dérivé.

Il n'est pas anodin qu'un historien du droit international comme Wilhelm Janssen avoue qu'il se sent dépaysé par les analyses de Schmitt. Les histoires du droit international de Reibstein et Nussbaum ${ }^{59}$ semblent plus normales à Janssen : elles parlent le langage familier du droit naturel et du droit positif. Mais dans l'enquête de Schmitt «tout tourne autour de termes comme Ordnung und Ortung, Landnahme, Raumordnung, globales Liniendenken ${ }^{60}$, etc. - termes que la littérature de l'histoire du droit international (Völkerrechtshistorie) ignore ou emploie en leur donnant un sens beaucoup plus restreint ${ }^{61}$. » Schmitt semble avoir transfiguré les lieux communs de la doctrine même du droit des gens. Janssen remarque d'ailleurs à juste titre «qu'une critique de la pensée schmittienne doit prendre comme point de départ sa conception des lignes d'amitié6 ${ }^{62} \gg$ et que la lecture que Schmitt propose de Vitoria ne peut se comprendre qu'une fois replacée dans l'ensemble des concepts clés du Nomos de la terre.

Nous disposons de certains indices de ce que le lieu d'où Schmitt se place pour réécrire l'histoire du droit international est la théorie moderne de l'impérialisme. Il semble en effet que Schmitt traduise, dans ses grandes lignes, la théorie marxienne de l'accumulation primitive du capital ou la théorie de Rosa Luxemburg et de Lénine - selon laquelle la structure politique de l'impérialisme est l'expression de la dépendance fondamentale du capitalisme vis-à-vis d'un monde non capitaliste - dans le langage de la science du droit international, et que c'est précisément cette importation d'une théorie étrangère au sein de la doctrine du droit international qui en dérangerait la terminologie et qui la rendrait méconnaissable aux spécialistes de la discipline.

Le premier indice qu'on peut relever est que le cinquième corollaire introductif du

${ }^{59}$ Arthur Nussbaum, Geschichte des Völkerrechts in gedrängter Darstellung, op. cit. ; Ernst Reibstein, Völkerrecht. Eine Geschichte seiner Ideen in Lehre und Praxis, 2 vol., Fribourg, Munich, Verlag Karl Alber, 1957-1963.

60 «Ordre et localisation», «prise de terres», « ordre spatial», «pensée selon les lignes globales ». Ces concepts sont difficiles à traduire précisément parce qu'ils sont des créations conceptuelles de Schmitt. Nous renvoyons au glossaire commenté de Peter Haggenmacher à la fin de l'édition française du Nomos de la terre.

${ }^{61}$ Wilhelm Janssen, ibid., p. 5

${ }^{62}$ Ibid. p. 6. 
Nomos de la terre sur « la prise de terres comme événement constituant du droit des gens » ébauche déjà des thèmes qui seront développés par la suite dans «Prendre/partager/paître » (1953), texte que Schmitt appelle d'ailleurs parfois «le septième corollaire du Nomos de la terre ${ }^{63}$ » et qui porte le sous-titre évocateur : «la question économique et sociale à partir du nomos». Or il se trouve que dans «Prendre/partager/paître », il n'est question que des théories politiques modernes. Il y est question de Marx, de Lénine, de Proudhon, et non de Vitoria, bien que les thèmes développés soient très proches de ceux du cinquième corollaire où n'apparaît que le nom de Vitoria. Dans son essai de 1953, Schmitt consacre un long passage à l'impérialisme selon Lénine ${ }^{64}$. On peut commencer par noter que dans ce texte, le style diffère grandement de celui auquel nous a habitués Le nomos de la terre. Au lieu de dire comme dans ce livre, dans un langage poétique mais vague, que tout nomos est précédé d'une prise, il écrit que «le partage et la production sont précédés de l'expansion impérialiste $^{65}$ ». Il parle « des prises de terres primitives des temps préindustriels ${ }^{66} »$, «des pillages ouverts du capitalisme primitif » et des «formes dissimulées de la prise sous lesquelles s'opère l'appropriation, par le capitaliste, de la plus-value produite par le travailleur $^{67}$ ». Le juriste reprend alors un thème qu'il avait traité plus en profondeur, quelques décennies plus tôt, dans Die geistgeschichtliche Lage des heutigen Parlementarismus, à propos de la différence entre Lénine et Marx. Il reproche à Marx et à Engels d'être encore trop métaphysiciens, trop attentistes - ils ne misent pas sur l'action directe - et trop rationalistes. Aux yeux de Schmitt, Marx et Engels continuent comme Dühring de «naturaliser » la violence et le pillage et de les insérer dans une chaîne de causes et d'effets, au lieu d'y voir comme Lénine des moments fragiles de crise qui sont la matière même de l'action politique, de son imprévisibilité, de sa précarité, et le point d'ancrage de la transformation de l'histoire par la politique. La «civilisation» des libéraux et la «lutte des classes » des marxistes se rejoignent en ceci, pour Schmitt, qu'elles représentent toutes deux la raison qui se révèle lentement à elle-même. Par rapport aux socialistes et aux libéraux qui pensent que pour résoudre la

${ }^{63}$ Carl Schmitt, Verfassungsrechtliche Aufsätze aus den Jahren 1924-1954, Berlin, Duncker \& Humblot, 2003 [1958], p. 501 ; Carl Schmitt, La guerre civile mondiale. Essais (1943-1978), trad. par Céline Jouin, Maisons-Alfort, ère, 2007, p. 62.

${ }^{64}$ Carl Schmitt, Verfassungsrechtliche Aufsätze aus den Jahren 1924-1954, op. cit., p. 495 ; Carl Schmitt, La guerre civile mondiale. Essais (1943-1978), op. cit., p. 56.

${ }^{65}$ Ibid.

${ }^{66}$ Carl Schmitt, Verfassungsrechtliche Aufsätze aus den Jahren 1924-1954, op. cit., p. 499 ; Carl Schmitt, La guerre civile mondiale. Essais (1943-1978), op. cit., p. 60.

${ }^{67}$ Ibid. 
question sociale, il suffit d'accroître la production ${ }^{68}$, Lénine paraît plus lucide à Schmitt. Lénine (et non Vitoria) est le grand théoricien de la «prise de terres ». Dans L'impérialisme, stade suprême du capitalisme (1916), Lénine montre que la guerre impérialiste moderne est une «guerre de conquête, de pillage, de brigandage ${ }^{69}$ ». Schmitt dit ouvertement dans son «septième corollaire » de 1953 qu'à ses yeux Lénine a le mérite d'avoir compris que le fait «que le partage et la production fussent précédés de l'expansion impérialiste, donc d'une prise et en particulier d'une prise de terres » était encore actuel dans le système-monde du $\mathrm{XX}^{\mathrm{e}}$ siècle et n'était pas un fait «moyenâgeux, pour ne pas dire atavique, réactionnaire ${ }^{70}$ ». L'idée essentielle est que le pillage n'est pas une affaire close : il continue même si le droit de conquête a été révoqué depuis longtemps. Le pillage ne se situe pas seulement au commencement de l'ère capitaliste mais il perdure à travers les «nouveaux partages » sociaux ${ }^{71}$, car, comme Schmitt le dira à Alexandre Kojève, on ne peut donner sans prendre. Il faut prendre aux uns pour donner aux autres : l'État social rétablit peut-être une certaine justice «à l'intérieur » mais il continue de piller la périphérie. Replacé dans le système mondial, l'État social européen est un privi-lège à proprement parler, une «loi privée ». Centrale est donc pour Schmitt l'idée que la prise perdure sous d'autres formes après l'ère des prises de terres coloniales, idée qu'on trouve déjà dans L'impérialisme, stade suprême du capitalisme ${ }^{72}$.

On peut penser que «Prendre/partager/paître », paru trois ans après Le nomos de la terre était déjà en gestation au moment où Schmitt rédigeait son livre, et qu'il a décidé de publier à part ses réflexions trop directement économiques, à tonalité trop léninistes. Mais il est vrai que le vocabulaire directement marxiste et économique de l'essai permet de décoder bien des passages du Nomos de la terre. On comprend soudain pourquoi Schmitt reproche à Grotius et Pufendorf de ne pas faire le lien entre la question générale de l'appropriation et la prise de terres mondiale dont ils sont pourtant les spectateurs, pourquoi il leur reproche de poser le problème de la propriété uniquement dans le cadre étroit et individualiste du droit civil. Ce lien, Schmitt l'établit lui-même constamment.

${ }^{68}$ Carl Schmitt, Verfassungsrechtliche Aufsätze aus den Jahren 1924-1954, op. cit., p. 495-496 ; Carl Schmitt, La guerre civile mondiale. Essais (1943-1978), op. cit., p. 56-57.

69 Vladimir Illich Lénine, L'impérialisme, stade suprême $d u$ capitalisme, Paris, Éditions sociales, 1979, p. 10.

${ }^{70}$ Carl Schmitt, Verfassungsrechtliche Aufsätze aus den Jahren 1924-1954, op. cit., p. 495 ; Carl Schmitt, La guerre civile mondiale. Essais (1943-1978), op. cit., p. 56.

${ }^{71}$ Carl Schmitt, Verfassungsrechtliche Aufsätze aus den Jahren 1924-1954, op. cit., p. 499 ; Carl Schmitt, La guerre civile mondiale. Essais (1943-1978), op. cit., p. 60.

${ }^{72}$ Cf. Lénine, ibid., p.125 sq. 
C'est même sa spécificité la plus nette par rapport aux autres internationalistes nonlibéraux de son temps, qui souvent restent pris dans la question de l'équilibre européen. Mais cette spécificité n'est pas une originalité absolue, c'est un apport des économistes, des marxistes, des théoriciens de l'impérialisme. Schmitt lit Grotius et Vitoria à partir de Marx et de la théorie de l'accumulation primitive. C'est pourquoi il trouve qu'il manque quelque chose dans Grotius, que Vitoria, selon lui, a vu. Il peut alors reprocher au juriste hollandais de n'avoir pas vu ce qu'il avait pourtant «sous les yeux ». Cela est manifeste quand il parle des lignes d'amitié. Contrairement à ce qu'affirme Schmitt, le principe «no peace beyond the line »n'a jamais été un grand principe de la dogmatique du droit des gens. S'il continue à évoquer ce principe censé venir du XVII siècle quand il parle de l'Acte du Congo de 1885, n'est-ce pas parce qu'il lui fait porter toute la charge de l'opposition entre capitalisme et monde précapitaliste, entre exploiteurs et exploités, qu'il tire non pas bien sûr de la dogmatique du droit des gens du XVII ${ }^{\mathrm{e}}$ siècle mais de la théorie marxiste?

Le dernier indice que nous relèverons est une note du Glossarium. Le juriste note dans son journal, le 26 février 1948 :

Excitants, les sept titres juridiques que donne Vitoria pour la conquête espagnole de l'Amérique. À lui seul, l'ordre dans lequel il les présente est étonnant dans la bouche d'un moine chrétien : jus commercii au début, jus praedicandi (propagande), jus protegendi Christianos conversos, jus Christiani principis a Papa delegato et instituto, jus interventionis contra tyrannidem, jus liberae electionis, jus protectionis socios! D'ailleurs les deux premiers, le commerce et la propagande, se fondent l'un dans l'autre et ils sont l'objet des $9 / 10^{\mathrm{e}}$ de l'argumentation. À la fin le gouvernement espagnol sera rassuré parce qu'il n'aura pas subi de pertes financières et parce que l'impérialisme des Romains et les titres chrétiens se sont révélés bien utiles, parce qu'il sera mis devant le fait accompli d'une christianisation déjà bien entamée qui justifiera toutes les guerres justes que l'on voudra [...]. Heureusement que cette aura de Vitoria n'a pas été analysée par Karl Marx ou par Lénine (Gut, daß dieser Vitoria-Nimbus nicht von Karl Marx oder von Lenin analysiert worden ist). Les choses se seraient corsées. Je me demande toujours: mais que veut-il au fond? Apparemment il ne veut rien changer à l'aspect politique et économique de 
la Conquista. Que veut-il donc ? Il veut rester maître de l'argumentation, pour conserver sa suprématie spirituelle (Er will die Argumentation in die Hand bekommen, um die geistige Führung zu behalten $)^{73}$.

On voit que la lecture de Vitoria à partir de Marx et de Lénine, qui est à notre avis une clé du Nomos de la terre, n'est pas une hypothèse si farfelue. Elle a été évoquée au moins une fois par Schmitt.

Il reste à se demander quelle est la logique de cette lecture de Vitoria en deux temps. Disons que nous ne l'examinons pas pour elle-même, mais parce qu'elle donne probablement la clé du discours de Schmitt sur la guerre juste. La question que certains se posent concerne un contresens que commet Schmitt, contresens qui ressort de l'architectonique d'ensemble de la théorie schmittienne de la guerre, mais que l'on trouve en fait assez rarement dans la lettre de son texte. Il s'agit de l'identification entre guerre juste et guerre totale, ou guerre juste et guerre sainte. On l'a dit, la théorie médiévale de la guerre juste refusait la guerre vindicative et déchaînée. Seule une guerre limitée pouvait être qualifiée de juste pour Vitoria, Grotius et les autres. La théorie de la guerre juste soumettait à certaines conditions formelles la violence guerrière, la justa causa n'étant qu'une de ces conditions, nullement suffisante. Or, cela, l'historien Schmitt le sait. Pour autant, l'impression perdure que Schmitt condamne le retour de la théorie de la guerre juste et y voit un retour de la guerre totale. Pourquoi Schmitt ne parle-t-il pas plutôt du retour de la guerre sainte ou du retour des croisades ?

Le 29 juin 1948, Schmitt recopie dans son journal le passage d'une lettre qu'il a envoyée à Günther Kraus :

Quand je songe à l'attitude de Vitoria et aux conséquences qu'elle a eues, je ne peux m'empêcher de trembler devant le dominicain. Je crains maintenant que mon chapitre sur Vitoria du Nomos [de la terre] soit beaucoup trop indulgent, pétri de scrupules et circonspect, et que par là même il perde de sa force. Ce que j'y affirme très discrètement sur Nys, James Brown Scott (et du coup aussi sur Jackson ${ }^{74}$ ) a pourtant au fond la même portée que ce qu'ont dit et fait Augustin Cochin, Vallery-Radot et le jeune Bernanos

\footnotetext{
${ }^{73}$ Glossarium, op. cit., p. 105-106.

${ }^{74}$ Robert Jackson avait été Attorney general puis juge de la Cour suprême des États-Unis avant de prendre la tête de l'accusation américaine à Nuremberg. Il était le supérieur de Robert Kempner, le juriste qui a interrogé Schmitt à Nuremberg.
} 
(avant qu'il ne s'endorme tout à fait). Mais qui l'entend? Pour les dominicains, Vitoria est un dieu domestique et un objet de prestige. Que peut-on faire en tant que profane? Ubi nihil vales, ubi nihil velis ${ }^{75}$. Je pourrais montrer à partir de Vitoria - et même en partant de ce qu'il dit luimême dans la cinquième dubia «circa bellum iustum » et la neuvième dubia « quantum liceat in bello iusto » - que la guerre juste est la guerre totale, mais qui cela intéresse-t-il ${ }^{76}$ ?

Bien que Schmitt écrive à Günther Kraus (dans son journal et en privé) qu'il pourrait montrer «à partir de Vitoria que la guerre juste est la guerre totale », il sait qu'historiquement c'est inexact. Ce n'est pas la guerre juste de Vitoria qui est une guerre totale, c'est la guerre impérialiste contemporaine. L'envie qu'il avait de le montrer en dépit des faits est peut-être l'un des facteurs qui donne à sa lecture de Vitoria son aspect embrouillé et sinueux.

En vérité, l'exactitude historique et philologique semble ne pas avoir été le seul objectif de Schmitt. Elle n'était qu'une étape de son démontage de l'humanisme théorique, l'étape du chapitre sur Vitoria, moment de la réfutation des thèses exagérées de James Brown Scott dont la motivation idéologique était si visible. La vacillation de Schmitt sur la guerre juste (parfois assimilée à la guerre totale, parfois distinguée de la guerre sainte) n'est pas une ignorance. Elle surgit au point de rencontre de deux exigences distinctes.

Dans le chapitre sur Vitoria du Nomos de la terre, Schmitt part à la recherche du «vrai » Vitoria pour montrer que les pacifistes et les doctrinaires de la SDN n'ont pas à se l'approprier comme ils le font. Toute son analyse vise à distinguer deux concepts de guerre juste: la conception authentique du théologien espagnol et ses déviations construites au XX $\mathrm{X}^{\mathrm{e}}$ siècle. Schmitt souligne l'écart ${ }^{77}$ entre les Relectiones et le Protocole de Genève de 1924 ou le pacte Briand-Kellogg de 1928. D’où les phrases du type : «la guerre juste du Moyen Âge admet une guerre juste d'agression », expression étonnante puisque la notion de guerre d'agression n'est apparue qu'au $\mathrm{XX}^{\mathrm{e}}$ siècle. La guerre juste contemporaine est pour Schmitt une «guerre juste positivée ». Les traités internationaux reposent «sur l'idéal d'une interdiction automatique de l'agression et sur le refus de

\footnotetext{
75 «Là où tu ne vaux rien, tu ne dois rien vouloir ».

${ }^{76}$ Glossarium, op. cit., p. 173.

${ }^{77}$ Le nomos de la terre, op. cit., p. 121.
} 
poser le problème de la guerre juste ${ }^{78}$ ». «Celui qui tire le premier coup de feu ou franchit la frontière en premier » devient celui qui commet une guerre injuste ${ }^{79} »$. C'est l'absence d'un concept de guerre véritable que le juriste regrette. L'agresseur est celui qui attaque en premier, principe qui est au service du statu quo.

En somme, dans le chapitre sur Vitoria, voulant montrer le «vrai » Vitoria, Schmitt présente la «méthode anhistorique », «à distance des faits » du théologien espagnol. En revanche, dans le second groupe de textes (le cinquième corollaire et la section sur Grotius et Pufendorf), Vitoria apparaît du côté de ceux qui «édifient une science du droit des gens qui a conscience de ses propres prémisses historiques ${ }^{80} \gg$. Dans le chapitre sur Vitoria, le concret est le biographique, le textuel, les intentions. Schmitt proteste contre le détournement d'autorité auquel procède assez grossièrement James Brown Scott et nous plonge, en philologue et en historien, dans ces détails qui font la substance même du livre du futur cardinal Joseph Höffner, détails que Schmitt trouve d'ailleurs souvent chez Höffner (Vitoria le directeur de conscience, la «situation concrète » du confesseur par rapport à celle du conseiller juridique ${ }^{81}$, etc.). Schmitt joue ainsi la carte du retour au sens originel puisque c'est comme un retour aux sources du droit international que les wilsoniens invoquent Vitoria. Provisoirement donc, Schmitt ne met pas en question le présupposé des libéraux : le sens authentique du texte de Vitoria doit donner accès à l'essence du droit international.

Mais dans le second groupe de textes, l' «origine»n'a plus le même sens. Elle désigne non plus le sens authentique des doctrines des premiers auteurs mais la prise de terres. Le concret n'est plus la biographie de Vitoria, dans ses détails, mais la Conquista elle-même. La vérité de la théorie de Vitoria ne surgit plus au détour d'une analyse interne du texte mais de la conscience que cette théorie a de ses «propres prémisses

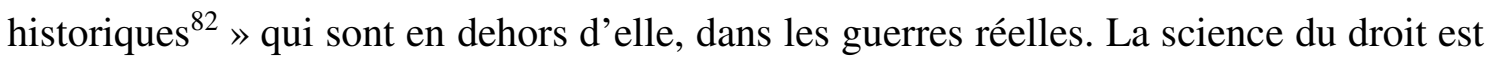
alors conçue comme une superstructure dérivée à partir d'une base réelle : la science vraie a conscience de sa provenance (Vitoria), l'idéologie est oubli de l'origine matérielle (Grotius et Pufendorf). La théorie de Vitoria est vraie parce qu'elle a

${ }^{78}$ Carl Schmitt, Entwicklung der internationalrechtlichen Pönalisierung des Angriffskrieges (1919-1939), Hauptstaatsarchiv de Düsseldorf, RW 265-19220, p. 22.

${ }^{79}$ Ibid., p. 23.

${ }^{80}$ Le nomos de la terre, op. cit., p. 137, p. 138.

${ }^{81}$ Ibid., p. 111.

${ }^{82}$ Ibid., p. 137. 
conscience de son propre caractère «constitué ${ }^{83} »$, dérivé, par rapport aux plus «constituantes »des guerres, les guerres coloniales. Le langage marxiste n'est pas employé mais sa syntaxe est présente. Vitoria n'est plus compris à partir de lui-même dans ce groupe de textes. Sa pensée y est insérée dans un ensemble de concepts qui ne viennent pas de lui. C'est la suture avec le reste du livre que le second groupe de textes opère. Et l'on parvient enfin à traduire certains des concepts de Schmitt les plus importants : nomos peut signifier «base », lignes d'amitié «rapport de dépendance entre monde capitaliste et monde préindustriel », prise de terres «impérialisme », etc. C'est bien la théorie marxienne de l'idéologie ainsi que la théorie de l'impérialisme qui sont constamment présupposées : Grotius et Pufendorf donnent pour Schmitt une vue superficielle du droit des gens car il s'en tiennent au droit bien réglé de l'Europe et oublient qu'il a pour condition la violence dans les colonies. C'est un coup de force de Schmitt, car pour Vitoria le droit des gens ne saurait être une «science» dont la question du droit des Indiens serait les «prémisses historiques ».

Finalement, dans le chapitre sur Vitoria aussi, la préoccupation première de Schmitt est l'actualité. Le long détour par l'exégèse des Relectiones est stratégique. Schmitt y oppose la théorie de la guerre juste de Vitoria à celle des Américains et des institutions internationales du $\mathrm{XX}^{\mathrm{e}}$ siècle, qui s'opposent l'une à l'autre comme la guerre limitée à la guerre totale. Dans le second groupe de textes, c'est la théorie de l'impérialisme et non la théorie médiévale de la guerre juste qui est centrale. La question de l'origine du système mondial prévaut. Schmitt oppose alors ensemble les deux théories de la guerre juste, l'ancienne et la nouvelle, à leurs conditions matérielles d'émergence. En tant qu'intellectuel européen favorisé, Vitoria, que veut-il ? demande-t-il dans son journal ${ }^{84}$. Et il répond : conserver sa suprématie intellectuelle (die geistige Führung behalten). Ce n'est pas intentionnellement ni dans son texte que Vitoria «justifie » la conquête, mais c'est le « résultat essentiel ${ }^{85}$ », l'effet réel, inconscient, anonyme, de la « vieille doctrine de la guerre juste », quand on critique en elle l'idéologie. Dans le second groupe de textes, Schmitt a systématiquement substitué le vocabulaire de l'effet inconscient au vocabulaire des intentions : «la théorie de la guerre juste de Vitoria permet la prise territoriale de sol étranger ${ }^{86} \ldots$ ». La théorie dans son ensemble devient universalité fictive et la différence entre guerre juste et guerre totale s'estompe ( «je pourrais

\footnotetext{
${ }^{83}$ Ibid., p. 85.

${ }^{84}$ Glossarium, op. cit., p. 106.

${ }^{85}$ Le nomos de la terre, op. cit., p.170.

${ }^{86}$ Ibid., p. 85.
} 
montrer à partir de Vitoria que la guerre juste est la guerre totale...). Il n'est plus gênant, dès lors, de considérer que la signification de l'œuvre de Vitoria est l'ensemble de ses réutilisations, construites au cours du temps, que celle-ci n'est ni achevable, ni réductible à un seul principe. D'ailleurs, Schmitt le disait déjà dans son chapitre sur Vitoria («Qu'on transporte des arguments à des situations nouvelles n'a en soi rien d'insolite et se produit souvent dans l'histoire $\left.{ }^{87} \ldots »\right)$. C'est par sa syntaxe et non pas son langage (théologique) que la théorie de Vitoria est vraie. Le langage de Grotius et de Pufendorf a beau être plus moderne, il est oublieux de la prise, qui reste impensée. Voilà peut-être la raison pour laquelle Schmitt s'intéresse à Vitoria plutôt qu'à Suarez : à ses yeux Vitoria est plus moderne que Suarez, bien que Suarez soit le véritable inventeur du droit interétatique moderne et que le jus gentium de Vitoria soit encore d'ascendance médiévale et théologique. C'est que le Vitoria de Schmitt remonte en deçà du jus inter gentes, plus proche de l'origine «matérielle » ou « réelle » qu'est la prise de terres violente. À cet égard on peut dire que Vitoria est le «fantasme » de Schmitt et l'on comprend qu'il soit son «grand thème », thème de la « dé-théologisation du droit des gens ». Dans le texte schmittien, Vitoria a pour fonction de combler le divorce entre idéal et réalité, divorce qui caractérise toute l'histoire doctrinale du droit des gens, vue du $\mathrm{XX}^{\mathrm{e}}$ siècle.

On comprend maintenant pourquoi les spécialistes du droit international comme August Friedrich von der Heydte ou Wilhelm Janssen ont pu être décontenancés par la lecture schmittienne de Vitoria : c'est qu'ils restaient eux-mêmes à l'intérieur de la tradition doctrinale du droit des gens et n'avaient pas l'idée d'aller chercher ailleurs les sources de Schmitt. Certains ont tout de suite compris en lisant les écrits du juriste, par exemple Carl Brinkmann ou François Perroux ${ }^{88}$. Mais ce sont des économistes.

\section{Recebido em janeiro de 2012 \\ Aceito em janeiro de 2012.}

\footnotetext{
${ }^{87}$ Ibid., p. 116.

${ }^{88}$ On se reportera à la recension que fait Carl Brinkmann de Positionen und Begriffe dans le Schmoller Jahrbuch, $\mathrm{n}^{\circ} 64$, cahier 4, mai 1940, Hauptstaatsarchiv de Düsseldorf, RW 265-21355 ainsi qu'à François Perroux, L'économie du XX siècle, Presses Universitaires de Grenoble, 1991, p. 545.
} 